\title{
AN EXAMPLE CONCERNING INVERSE LIMIT SEQUENCES OF NORMAL SPACES
}

\author{
M. G. CHARALAMBOUS
}

\begin{abstract}
Using techniques developed by Wage and Przymusiński, we construct an inverse limit sequence $\left(X_{n}, f_{n m}\right)$ with limit space $X$ such that each $X_{n}$ is Lindolof with $\operatorname{dim} X_{n}=0$, where $\operatorname{dim}$ denotes covering dimension, while $X$ is normal with $\operatorname{dim} X>0$. The space $X$ is a counterexample to several conjectures in Topology.
\end{abstract}

Let $X$ be the inverse limit of the sequence $\left(X_{n}, f_{n m}\right)$. We recall that if, for some $k$ in $N$, the set of positive integers, $\operatorname{dim} X_{n}<k$ for each $n$ in $N$, then $\operatorname{dim} X<k$ if one of the following conditions is satisfied: (a) $X_{n}$ is perfectly normal for each $n$ in $N$, (b) $X$ is strongly paracompact, and (c) $X$ is countably paracompact, $X_{n}$ is normal and $f_{n m}$ is open and surjective for all $n, m$ in $N$ [1], [4].

The Construction. Let $d$ be the usual metric on the unit interval $I$. Let $\rho$ be the separable metric on $I$ introduced by Wage [8]. The $\rho$-topology on $I$ is finer than the $d$-topology, $d<\rho$ and every $\rho$-Borel set of $I$ is also $d$-Borel. Moreover, every nonempty $\rho$-open set is uncountable and there is an $\rho$-closed set $E$ such that the boundary of every nonempty $\rho$-open set disjoint from $E$ has cardinality $c$, the cardinality of the continuum.

Let $\left\{A_{1}, A_{2}, \cdots\right\}$ be a partition of $I$ with the property that for every uncountable Borel set of $B$ of $I$ and every $n$ in $N,\left|B \cap A_{n}\right|=c$ [6, Theorem 2]. We may clearly assume that 0,1 are in $A_{1}$ and that $A_{1} \cap E$ is $\rho$-dense in $E$. For each $x$ in $I-A_{1}$ and each $n$ in $N$, choose $x_{n}^{-}, x_{n}^{+}$in $A_{2}$ so that

$$
x-\frac{1}{n}<x_{n}^{-}<x_{n+1}^{-}<x<x_{n+1}^{+}<x_{n}^{+}<x+\frac{1}{n} \text {. }
$$

Let $\left\{\left(A_{\alpha}, B_{\alpha}\right): \alpha<\omega(c)\right\}$ be the collection of all pairs of countable subsets of $A_{1}^{2}$ with $\left|\overline{A_{\alpha}^{\rho}} \cap \overline{B_{\alpha}^{\rho}} \cap \Delta\right|$ uncountable, where $\omega(c)$ is the first ordinal of cardinality $c$ and $\Delta$ is the diagonal of $I^{2}$. For each $\alpha<\omega(c)$ and each $n$ in $N$, choose $x_{\alpha}$ in $A_{1}$, $\left(x_{\alpha n}^{1}, x_{\alpha n}^{2}\right)$ in $A_{\alpha}$ and $\left(x_{\alpha n}^{3}, x_{\alpha n}^{4}\right)$ in $B_{\alpha}$ so that $\rho\left(x_{\alpha n}^{i}, x_{\alpha}\right)<1 / n, x_{\alpha n}^{i} \triangleleft x_{\alpha}$ and $x_{\beta} \triangleleft x_{\alpha}$ for $\beta<\alpha$ and $i=1,2,3,4$, where $\triangleleft$ is a well-ordering on $I$.

For $n, m$ in $N$ and $x$ in $I$, we define a set $B_{m}^{n}(x)$ containing $x$ as follows. For $x$ in $A_{2} \cup \cdots \cup A_{n+1} \cup\left(A_{1}-\left\{x_{\alpha}: \alpha<\omega(c)\right\}\right), B_{m}^{n}(x)=\{x\}$. For $x$ in $\cup_{k=n+2}^{\infty} A_{k}$, $B_{m}^{n}(x)=\left[x_{m}^{-}, x_{m}^{+}\right]$. On $\left\{x_{\alpha}: \alpha<\omega(c)\right\}, B_{m}^{n}$ is defined by transfinite induction.

Assuming it has been defined for all $\beta<\alpha$, we set

$$
B_{m}^{n}\left(x_{\alpha}\right)=\left\{x_{\alpha}\right\} \cup\left(B_{k+2 m}^{n}\left(x_{\alpha k}^{i}\right): k>2 m, i=1,2,3,4\right) .
$$

Received by the editors July 6, 1978 and, in revised form, March 2, 1979.

AMS (MOS) subject classifications (1970). Primary 54G20, 54F45.

$K e y$ words and phrases. Normal, Lindelö, paracompact, topologically complete and $N$-compact space, covering dimension. 
It is readily seen that $B_{m}^{n}(x)$ is a $d$-closed subset of $[x-1 / m, x+1 / m]$ and $B_{m}^{n}\left(x_{\alpha}\right)$ is a countable subset of $\left\{x: x \triangleleft x_{\alpha}, \rho\left(x, x_{\alpha}\right)<1 / m\right\}$. Also $B_{m+1}^{n}(x) \subset$ $B_{m}^{n}(x)$ and if $y$ is in $B_{m}^{n}(x)$, then $B_{k}^{n}(y) \subset B_{m}^{n}(x)$ for some $k$ in $N$. It follows that $\left\{B_{m}^{n}(x): m \in N\right\}$ is a local base at $x$ consisting of open-and-closed sets relative to some topology $\tau_{n}$ on $I$. Next, put $B_{m}(x)=B_{m}^{n}(x)$ if $x=x_{\alpha}$ for some $\alpha<\omega(c)$, and $B_{m}(x)=\{x\}$ if not. $B_{m}(x)$ is a local base of $x$ consisting of open-and-closed sets relative to some topology $\tau$ on $I$. Clearly, $\tau$ is finer than the $\rho$-topology on $I$, and $\left\{x_{\alpha n}^{i}: n=1,2, \ldots\right\}$ converges to $x_{\alpha}, i=1,2,3,4$.

In the sequel, $X, X_{n}$ denote $(I, \tau),\left(I, \tau_{n}\right)$, respectively, and $f_{n m}: X_{m} \rightarrow X_{n}$ denotes the identity function. It is readily verified that $X$ is the limit space of the inverse limit sequence $\left(X_{n}, f_{n m}\right)$.

Claim 1. $X_{n}$ is a $T_{2}$ Lindelöf space with $\operatorname{dim} X_{n}=0$.

Proof. It is obvious that $X_{n}$ is $T_{2}$ and ind $X_{n}=0$. Let $थ$ be an open cover of $X_{n}$. Since clearly every point of $A_{n+2}$ has a local base consisting of open intervals, there are $d$-open sets $G_{1}, G_{2}, \ldots$, each contained in some member of $\mathscr{U}$, such that $A_{n+2} \subset G=\cup_{i=1}^{\infty} G_{i}$. Then, since $(X-G) \cap A_{n+2}=\varnothing, X-G$ is countable. It is now clear that $\mathcal{Q}$ has a countable open refinement, and hence $X_{n}$ is Lindelöf. Thus, since also ind $X_{n}=0, \operatorname{dim} X_{n}=0$.

ClaIM 2. The family of neighbourhoods of the diagonal of $X^{2}$ is a uniformity. Hence $X$ is normal [2, Problem L, p. 209].

Proof. Let $G$ be an open neighbourhood of $\Delta$. It suffices to find a neighbourhood $V$ of $\Delta$ with $V \circ V \subset G$.

Let $A=A_{1}^{2}-G$. If $\bar{A}^{\rho} \cap \Delta$ were uncountable, then, for some $\alpha<\omega(c), A$ would contain $A_{\alpha}=B_{\alpha}$ as a countable $\rho$-dense subset and hence $\left(x_{\alpha}, x_{\alpha}\right)$ would be a point of $A$. Thus, $\bar{A}^{\rho} \cap \Delta$ is countable. Since $\rho$ is separable and $X^{2}$ is Tychonoff, there is a cozero cover $\left\{G_{1}, G_{2}, \ldots\right\}$ of the open-and-closed subset $A_{1}$ of $X$ such that

$$
\Delta \cap A_{1}^{2} \subset \bigcup_{i=1}^{\infty} G_{i}^{2} \subset G .
$$

Let $\left\{H_{1}, H_{2}, \ldots\right\}$ be a cozero star-refinement of $\left\{G_{1}, G_{2}, \ldots\right\}$ and put $V=$ $\cup_{i=1}^{\infty} H_{i}^{2} \cup \Delta$. It is readily verfied that $V$ has the required properties.

Claim 3. $\operatorname{dim} X>0$.

Proof. Let $F$ be an uncountable $\rho$-closed set of $I$ with $E \cap F=\varnothing$, and suppose $U, V$ are disjoint open-and-closed sets of $A_{1}$ with $E \cap A_{1} \subset U, F \cap A_{1} \subset V$ and $A_{1}=U \cup V$. Then $V$ is uncountable and $\bar{U}^{\rho} \cap \bar{V}^{\rho}$ is countable, otherwise, for some $\alpha<\omega(c), A_{\alpha}, B_{\alpha}$ would be countable $\rho$-dense subsets of $U^{2}, V^{2}$, respectively, and hence $x_{\alpha}$ would be in $U \cap V$. Also $\bar{U}^{\rho} \cup \bar{V}^{\rho}=\bar{A}_{1}^{\rho}=X$ and, since $A_{1} \cap E$ is $\rho$-dense in $E, E \subset \bar{U}^{\rho}$. Hence $X-\bar{U}^{\rho}=\bar{V}^{\rho}-\bar{U}^{\rho}$ is an uncountable $\rho$-open set of $I$ contained in $X-E$ with $\rho$-boundary contained in the countable set $\bar{U}^{\rho} \cap \bar{V}^{\rho}$. We conclude that $\operatorname{dim} A_{1}>0$ and hence $\operatorname{dim} X>0$.

REMARK 1. A space is called $N$-compact if it is the inverse limit of countable discrete spaces. A zero-dimensional $T_{2}$ Lindelöf space is $N$-compact, and so is the inverse limit of $N$-compact spaces. Hence $X$ is $N$-compact although $\operatorname{dim} X>0$. 
Przymusiński's space in [7] has the same property. Spaces exhibiting the same pathology were previously constructed in [3] and [5].

REMARK 2. Kelley [2, p. 209] has conjectured that a topologically complete space with the property that the family of all neighbourhoods of its diagonal is a uniformity is paracompact. $X$ is a counterexample to this conjecture. For if $X$ were paracompact, since it is also locally countable, we would have $\operatorname{dim} X=\operatorname{loc} \operatorname{dim} X$ $=0$.

\section{REFERENCES}

1. M. G. Charalambous, The dimension of inverse limits, Proc. Amer. Math. Soc. 58 (1976), 289-295.

2. J. L. Kelley, General topology, Van Nostrand, Princeton, N.J., 1955.

3. S. Mrowka, Recent results on E-compact spaces, Proceedings of the Second Pittsburgh International Conference, Lecture Notes in Math., vol. 378, Springer-Verlag, Berlin and New York, 1974, pp. 298-301.

4. K. Nagami, Countable paracompactness of inverse limits and products, Fund. Math. 73 (1971), 261-270.

5. E. Pol and R. Pol, A hereditarily normal strongly zero-dimensional space with a subspace of positive dimension and an N-compact space of positive dimension, Fund. Math. 97 (1977), 43-50.

6. T. Przymusiński, On the notion of n-cardinality, Proc. Amer. Math. Soc. 9 (1978), 333-338.

7. On the dimension of product spaces and an example of M. Wage, Proc. Amer. Math. Soc. 76 (1979), 315-321.

8. M. Wage, The dimension of product spaces, Proc. Nat. Acad. Sci. U.S.A. (to appear).

Department of Mathematics, Ahmadd Bello University, Zaria, Nigeria

Current address: Department of Mathematics, University of Nairobi, Nairobi, P. O. B.30197, Kenya 